\title{
Tobacco, Alcohol, and Caffeine Use during Pregnancy
}

\author{
LAUREN S. AARONSON, RN, PHD, AND CAROL L. MACNEE, RN, MSN
}

\begin{abstract}
Research on the effects of smoking, alcohol, and caffeine on the outcomes of pregnancy is reviewed. The strength of the evidence on the effects of these substances is varied, ranging from some clear and consistent findings for smoking to inconsistent findings for caffeine. Evidence regarding alcohol is strong in some areas and less consistent in others. The implications for clinical practice, particularly that effects vary with both the timing and the amount of the substances used, are discussed.
\end{abstract}

Substantial evidence exists to support a causal relationship between the health and health behaviors of pregnant women and the subsequent health of their infants. The use of tobacco, alcohol, and caffeine are three behaviors for which evidence of the adverse effects of these substances on pregnancy outcomes is increasing. Research on the effects of these three substances has been extensive, multidisciplinary, and at times inconsistent. This inconsistency reflects the state of knowledge, as well as differences in the methods used and the controls applied in the various studies. Because nurses frequently intervene to promote positive maternal behaviors, an understanding of this diverse research and its clinical implications is crucial.

\section{SMOKING DURING PREGNANCY}

An estimated $20-30 \%$ of American women of childbearing age smoke despite clear and consistent research evidence on the adverse effects of smoking on pregnancy outcomes. ${ }^{1}$ In a landmark paper in 1957, Simpson first reported the association between maternal smoking and low birth weight. ${ }^{2}$ Since that time,

Accepted: September 1988 numerous studies have documented the adverse effects of maternal smoking. ${ }^{3-6}$

\section{Smoking and Low Birth Weight}

Smoking is a contributing factor in $20-40 \%$ of the cases of low birth weight infants in the United States and in general is associated with a 150-200 g reduction in infant birth weight. ${ }^{7}$ Mochizuki et al. found that infants born to women who smoked more than 20 cigarettes per day weighed on average $290 \mathrm{~g}$ less than infants of nonsmokers. ${ }^{3}$ In a prospective study of more than 800 Norwegian women, nearly one-half of the infants of smokers were in the lowest quartile for birth weight - an effect related to the number of cigarettes smoked. ${ }^{4}$ Blood thiocyanate levels (a physiologic measure of smoking behavior) have been negatively correlated with birth weights. ${ }^{5}$

Whereas smoking is associated with premature births, not all smoking-related low birth weight cases are attributable to prematurity. ${ }^{8}$ For example, despite equal maternal weight-for-height indices between smokers and nonsmokers, $60.7 \%$ of small-for-date infants had mothers who smoked, whereas only $29.2 \%$ average-for-date infant and $15.7 \%$ large-for-date infants had mothers who smoked. ${ }^{6}$ Further, smoking outcomes are dose-related. The odds of a woman delivering an infant weighing less than $2,500 \mathrm{~g}$ increases 
by $26 \%$ for every five cigarettes she smokes per day. ${ }^{9}$ Lastly, women's cessation of smoking during pregnancy has been demonstrated to increase birth weights. ${ }^{10}$

\section{Smoking is a contributing factor in $20-40 \%$} of the cases of low birth weight infants in the United States.

Results linking smoking to low birth weight are consistent even if smoking is grossly defined and persist despite reported reductions in the tar and nicotine contents in cigarettes. ${ }^{11,12}$ The relationship between smoking and low birth weight also holds across social classes, age groups, ethnic groups, and geographic locations. ${ }^{11}$ However, the extent of the effects attributed to smoking that may be caused by differences in other risk factors, such as age and previous pregnancy outcome, varies. ${ }^{7,13}$ For example, in a prospective clinical study, maternal age alone had no effect on birth weight, but among smokers, a reduction in birth weight was more pronounced with increasing maternal age. ${ }^{14}$

\section{Mechanisms of the Action of Smoking}

The physiologic effects of smoking remain speculative. Evidence exists that smoking reduces placental blood flow. ${ }^{15}$ Further, plasma volume is $25 \%$ less in pregnant women who smoke, which leads to artificially elevated hematocrits. ${ }^{16}$ Other researchers have found that the intervillous spaces of the placentae are larger in smokers and that serum concentrations of human placental lactogen (HPL), an indicator of the metabolic activity of the placenta, are lower in smokers. ${ }^{3}$ Changes and abnormal findings in the placentae of smokers include toxicity caused by elements in tobacco smoke, and placental ischemia as a consequence of nicotine-induced constriction of the uterine vessels. ${ }^{17}$

Specifically, smoking may affect birth weight by direct interference with nutrition through the physiologic depression of maternal weight gain or indirect interference through decreased maternal nutritional intake. Deibel suggests that smoking causes inadequate nutrition directly through difficulties in absorption and/or metabolism of calcium, vitamin $B_{12}$, vitamin $C$, and possibly vitamins $B_{6}, B_{1}$, and $A .{ }^{18}$ Deibel proposed that the fetus may be compromised from poor nutrient assimilation rather than from the toxic effects of tobacco alone. Rush found that smokers gained significantly less weight than nonsmokers, despite nutritional supplementation in both groups, and that smoking and maternal weight gain accounted for the greatest amount of variance in birth weight. ${ }^{19}$ Rush concluded that the effect of smoking on birth weight may be attributable to both a direct nutritional effect that causes a decrease in maternal weight gain and an indirect nutritional effect from caloric restriction.

Other researchers argue that a simple nutritional cause for growth retardation in the infants of smokers is unlikely. For example, the infants of smokers weigh less than the infants of nonsmokers, even when maternal weight gain is controlled. ${ }^{20}$ In a prospective study of smoking and dietary habits, mean caloric intake and weight gain were highest among smokers and lowest among nonsmokers. ${ }^{21}$ Further, the histologic, morphologic, and biochemical changes that occur in the placentae of smokers persist despite high protein supplementation. ${ }^{22}$ Thus, smoking affects birth weight separately from nutrition, and maternal weight gain does not counteract the effects of smoking.

\section{Other Effects of Smoking on Pregnancy Outcomes}

Whereas smoking clearly is implicated as a contributor to low birth weight, other effects of smoking on the outcomes of pregnancy are less clear. In a review of the effects of smoking on 28 pregnancy outcome variables (e.g., bleeding, premature rupture of membranes, stillbirths), Mclntosh found a "remarkable" conformity of relative risk factors and concluded that $15-45 \%$ of unfavorable pregnancy outcomes may be caused by smoking. ${ }^{23}$

\section{The Effects of Smoking on Perinatal Mortality}

Some studies report inconclusive results regarding smoking and perinatal mortality. ${ }^{7}$ This inability to obtain conclusive results may be because mortality rates are relatively low and large samples are necessary to demonstrate significant effects. ${ }^{24}$ On the other hand, several large studies have found that smoking clearly is associated with perinatal mortality. ${ }^{7,13,25}$ For example, the Ontario Perinatal Mortality Study, which used a sample of more than 50,000 single births, found strong evidence supporting a link between smoking and perinatal mortality. This study also found that perinatal mortality was dose-related and compounded by other risk factors. ${ }^{13}$ Women who smoked less than 20 cigarettes per day and had few other risk factors were at a $10 \%$ greater risk than nonsmokers, while women who smoked more than 20 cigarettes per day and had many associated risks were at $70-100 \%$ greater risk for losing their infants. ${ }^{13}$

\section{Congenital Abnormalities Secondary to Smoking}

Smoking has been implicated as a cause of congenital anomolies, ${ }^{26}$ decreased mental and behavioral 
development, ${ }^{27}$ and lower one-minute and five-minute Apgar scores. ${ }^{28}$ However, the results from studies of congenital abnormalities associated with maternal smoking are inconsistent, perhaps because of methodologic and analytic differences in the studies ${ }^{29}$ For example, several studies have found that mothers of infants with oral clefts smoked more than the mothers of infants with other birth defects. ${ }^{30,31}$ An increased incidence of congenital anomalies, primarily inguinal hernia and strabismus among male offspring, also has been reported for women who smoked more than 20 cigarettes per day. However, overall differences in the incidence of congenital anomolies between all smokers and nonsmokers were not statistically significant. ${ }^{26}$

\section{As many as $45 \%$ of all unfavorable pregnancy outcomes may be caused by smoking.}

Small, relatively mild cognitive abnormalities (e.g. hyperactivity, shorter attention span, and lower reading and spelling scores) also have been found in the children of mothers who smoked during pregnancy. ${ }^{27}$ Others have found attention and reaction time to be significantly related to smoking during pregnancy. ${ }^{32}$ Because both studies controlled for many confounding variables, their results warrant careful consideration.

While growth rate, height, and weight do not appear to be related to maternal smoking, anthropometric measures of adipose in children from 6 to 11 years of age have been found to be affected significantly by maternal smoking; thus, children of smokers have less adipose than do the children of nonsmokers. ${ }^{33}$ Additionally, for children maternal smoking has been implicated as a subsequent risk factor for the development of leukemias, lymphomas, and Wilms's tumor. ${ }^{34}$

Lastly, a more than threefold lowering of one- and five-minute Apgar scores has been demonstrated in the infants of mothers who smoked from 41 to 60 cigarettes per day. ${ }^{28}$ However, these results were not replicated in a similar but smaller study, so these results must be viewed with caution.

\section{The Clinical Implications}

The clinical implications for the findings described include the obvious need to encourage women not to smoke during pregnancy. Because the adverse effects of smoking are potentially dose-related and cumulative, nurses need to encourage and support cessation at any point during pregnancy. Even a decrease in smoking, and/or cessation in the last trimester, may improve pregnancy outcomes. Further, women with other risk factors for low birth weight and perinatal mortality should be particularly targeted for assistance with changing their smoking behaviors. Because the associated risk factors have been found to increase the risks of smoking, any potential modification of the associated factors also should be considered and facilitated, whether or not these women cease smoking.

\section{ALCOHOL USE DURING PREGNANCY}

The evidence on the effects of alcohol on fetal outcomes is murky, whereas that of smoking is quite clear. The literature on the ingestion of alcohol strongly suggests that drinking during pregnancy is associated with teratogenic effects and low birth weight. ${ }^{35-40}$ However, studies vary as to both the amount and the timing of alcohol ingestion that produce these effects. For example, although birth weight generally decreases with increased alcohol consumption, ${ }^{41}$ some investigations have found that this relationship holds only for heavy drinkers. ${ }^{4-43}$ Results are further complicated by the differing definitions of what constitutes heavy, moderate, and light drinking, as well as differences in the measures used (e.g., absolute alcohol versus the number of drinks).

\section{Effects of Alcohol on Birth Weight}

In a prospective study of more than 9,000 pregnant women, investigators found increased still births, low birth weight infants, and lower placental weights for infants born to mothers who consumed in excess of $1.6 \mathrm{oz}$ of absolute alcohol per day. ${ }^{45}$ The risk for these deleterious effects remained even after controlling for other risk factors such as smoking. The highest risk was associated with beer, rather than wine or liquor, despite the lower actual absolute alcohol content of beer. ${ }^{44}$

The deleterious effects of alcohol probably are exerted independently of the mother's nutritional status, despite the pattern of poor nutrition often associated with alcoholism. ${ }^{45}$ In laboratory studies, alcohol suppressed both maternal and fetal weight gain in rats fed low protein, protein adequate, or enriched diets. ${ }^{46}$

Alcohol-induced differences in infant birth weights are attributed to intrauterine growth retardation because alcohol consistently has been associated with low birth weight, even when gestation is controlled. ${ }^{35}$ Russell found twice as many very low birth weight infants born to mothers with alcohol-related psychi- 
atric diagnoses as compared to mothers with psychiatric diagnoses not related to alcohol. Further, three times the expected number of infants of mothers with alcohol-related psychiatric diagnoses experienced retarded intrauterine growth. ${ }^{38}$

\section{Alcohol deleteriously affects both birth weight and fetal development, and no safe levels of alcohol ingestion have been established.}

Although six drinks per day ( $3 \mathrm{oz}$ of absolute alcohol) constitute a major risk no safe levels of alcohol ingestion have been established. ${ }^{47,48}$ In one study on moderate drinking, daily consumption of $1 \mathrm{oz}$ of absolute alcohol in late pregnancy was associated with a $69 \mathrm{~g}$ greater decrease in birth weight than occurred when the same amount was consumed early in pregnancy ${ }^{36}$ Although this is a small difference individually, collectively this difference creates a downward shift in the distribution of weights for all infants. Consequently, more infants of mothers who drink will fall into the low birth weight category and be at risk for complications associated with low birth weight. In addition, infants born to women who are heavy drinkers but who reduced alcohol consumption before the third trimester have outcomes similar to those of infants born to women who are rare and moderate drinkers. ${ }^{41}$ Therefore, the timing of alcohol ingestion, not just the amount, can influence outcomes.

\section{Other Effects of Alcohol on Fetal Outcomes}

The most serious adverse effect associated with alcohol is the fetal alcohol syndrome (FAS). The characteristic features of FAS are consistently related to the extreme use of alcohol during pregnancy, particularly among alcoholic women. ${ }^{49,50}$ One of three infants born to alcoholic mothers has FAS. ${ }^{51}$ Fetal alcohol syndrome is not associated with single binges but occurs only when pregnant women consistently drink more than $80-100 \mathrm{~g}(2.8-3.5 \mathrm{oz})$ of alcohol daily. ${ }^{52}$

The effects of maternal alcoholism during pregnancy go beyond birth. At seven years of age, children born with FAS exhibit signs of borderline retardation or are frankly mentally retarded. ${ }^{50}$ Thus, the future mental defects of infants born to alcoholic mothers is as much of a concern as the effects noted at birth. ${ }^{53}$
The infants of nonalcoholic mothers who drink during pregnancy also may experience growth and neurologic effects that extend beyond birth. One study examined 453 infants of nonalcoholic mothers at birth and again at eight months and found that the average amount of alcohol consumed by these mothers during pregnancy was significantly related to lower infant weight and length at eight months of age. ${ }^{39}$ Among the neurological effects reported in infants are tremors, hand-to-face self-stimulation, an atypical head-to-left positioning, and other lower level motor activity. ${ }^{54}$ Other researchers report difficulties in state regulation, such as increased time to get to sleep and to awaken and abnormal patterning of sleep stages. ${ }^{55}$ In another study, $32 \%$ of the infants born to heavy drinkers (more than $1.5 \mathrm{oz}$ daily) demonstrated congenital abnormalities as compared to nine percent of the infants of abstinent mothers and $14 \%$ of those whose mothers were moderate drinkers. ${ }^{56}$

Despite substantial support for the negative effects of drinking on fetal and infant development, some contradictory evidence exists. For example, in a study of 12,400 women interviewed at the time of delivery, no association was found between the number of drinks per week and the overall rate of congenital malformations. ${ }^{57}$ Similarly, no congenital malformations were found among infants born to women with alcohol-related psychiatric diagnoses despite the infants' lower birth weights. ${ }^{38}$

\section{Possible Mechanisms of the Actions of Alcohol}

Clearly, alcohol deleteriously affects fetal development and birth weight. ${ }^{43,45}$ However, the amount of alcohol needed to cause these negative consequences and the mechanisms by which they occur remain unknown. Weiner and Rosett speculate that the adverse effects of alcohol are caused by the cumulative action of high concentrations of alcohol in the maternal-placental-fetal system, causing multiple alterations in the biochemical and physiologic processes. ${ }^{41}$ This variability of effects may be related to high blood alcohol concentrations at different stages of fetal development. In the first trimester, alcohol may affect the cell membrane and alter the embryonic organization of tissue. Throughout pregnancy, alcohol may interfere with the metabolism of carbohydrates, lipids, and proteins, and thus retard cell growth and division. Lastly, the central nervous system probably is most vulnerable in the third trimester, during which time rapid brain growth occurs. If heavy drinking ceases, the risk of further damage is reduced, and physiologic restitution and modifica. 
tion of abnormalities secondary to impaired growth may occur. ${ }^{41}$

Because alcohol passes easily through the placental barrier, concentrations found in the fetus are at least as high as those in the mother. Thus, the rate at which alcohol is metabolized may affect the amount of fetal damage. ${ }^{51}$ Further, susceptibility to these effects may be genetic, or may depend on prepregnancy weight, parity, and possibly age. ${ }^{49}$ Therefore, results remain unclear as to which is critical in predicting outcomes: the duration of drinking or the interaction of drinking with other factors. ${ }^{49}$

\section{Clinical Implications}

Because the exact mechanism by which alcohol affects pregnancy outcomes and the roles of the amount and timing of alcohol ingestion are not clearly understood, clinical interpretation is controversial. Strong arguments are advanced for both total abstinence and moderation. ${ }^{42,58}$ Specifically, more than one and one-half ounces of absolute alcohol (three to four drinks) per day will have a deleterious effect, and moderate drinking (defined as anywhere from daily to monthly) may have negative effects. Moreover, decreasing alcohol intake during pregnancy has lessened some effects. ${ }^{55}$ Therefore, nurses should err on the safe side clinically and advise and facilitate total abstinence from alcohol throughout pregnancy. If this seems unlikely or unreasonable for some women, decreasing alcohol consumption, particularly in the third trimester, is likely to improve outcomes. While assessment and support for behavior change need to begin at the first prenatal visit, continuing such efforts throughout the woman's pregnancy is equally important and valid.

\section{CAFFEINE USE DURING PREGNANCY}

In contrast to the effects of alcohol and tobacco, the effects of caffeine on pregnancy outcomes are the least conclusive and least consistent. At some dosages, caffeine has been found to be an animal teratogen. ${ }^{59,60}$ However, other studies report inconsistent results (see Brooten and Jordan for a review) ${ }^{61}$ Research findings that directly link caffeine to teratogenic effects in humans are limited, but some evidence exists. ${ }^{62}$

\section{The Teratogenic Effects of Caffeine in Humans}

Researchers conducting animal studies to demonstrate the teratogenic effects of caffeine used dosages of more than $80 \mathrm{mg} / \mathrm{kg}$. Fetal loss, decreased weight and size, and major skeletal defects were found. ${ }^{60}$ However, dosages as high as $80 \mathrm{mg} / \mathrm{kg}$ are substantially above pharmacologic levels and, in humans, would require drinking more than 45 cups of coffee per day. Moreover, in one study pregnant rats given either caffeinated or decaffeinated fluids gave birth to rat pups with similar physical and behavioral defects. This suggests that the teratogenic effects may be the consequence of some unidentified substance(s) other than caffeine. ${ }^{59}$

I Caffeine's half-life triples during pregnancy because of impeded caffeine clearance during this time. High quantities of caffeine may negatively affect pregnancy outcomes.

Human epidemiologic studies have yielded inconsistent results that have not supported the suspected teratogenic effects of caffeine. An increased incidence of spontaneous abortions, stillbirths, and premature births has been associated with maternal consumption of more than $600 \mathrm{mg}$ of caffeine per day (approximately eight cups of coffee).$^{62}$ However, these findings have not been replicated with larger samples.

For example, Linn et al. found no relationship between low birth weight, gestation, or malformations and drinking four or more cups of coffee per day. However, the researchers did not consider other sources of caffeine, such as cola or tea, and they did not control for changes in behavior during pregnancy. ${ }^{63}$ Further, no differences in maternal coffee consumption were found in more than 700 matched pairs of women who had children with and without congenital malformations ${ }^{64}$ Nonetheless, despite the little evidence supporting a suspected teratogenic effect, most investigators recommend that pregnant women avoid caffeine until more conclusive evidence is found. . $^{60,61,65,66}$

Additional findings from these studies have important clinical implications. For example, because caffeine equilibrates rapidly across the placenta, maternal concentrations of caffeine are good indicators of fetal exposure. Also, the half-life of caffeine triples during the third trimester of pregnancy ${ }^{66}$ Consequently, the same amount of caffeine results in much higher blood levels in the last trimester. Women who normally drink 10 cups of coffee per day, could approach pharmacologic plasma levels of caffeine (i.e. $11 \mathrm{mg} / \mathrm{kg} /$ day) during their last trimesters. 
The adverse effects of these substances are potentially dose-related and cumulative, underscoring a need to encourage and support cessation of their use at any point in pregnancy.

\section{Clinical Implications}

The clinical implications extracted from the literature on caffeine use during pregnancy are less clear than those for alcohol and tobacco. However, in very high quantities, caffeine may negatively affect pregnancy outcomes. Caffeine clearance decreases during pregnancy, potentially increasing exposure of the fetus to caffeine. Therefore, pregnant women should be advised to avoid caffeine as much as possible and should be informed about the common sources of caffeine, such as colas, other soft drinks (e.g., Mountain Dew, Dr. Pepper), chocolate, tea, and over-thecounter drugs. Further, women with a history of high caffeine intake should be particularly targeted for assistance with changing their behaviors, and warned that the amount of caffeine that remains in their systems, and therefore reaches their fetuses, will triple by the last trimester of pregnancy.

\section{THE COMBINED EFFECTS OF ALCOHOL, TOBACCO, AND/OR CAFFEINE}

No systematic studies of the co-occurrence of alcohol, tobacco, and caffeine use during pregnancy have been reported. However, a review of community studies supports a general co-occurrence of these substances. ${ }^{67-69}$ Smokers report greater alcohol and coffee drinking than do nonsmokers, and alcohol users report more coffee drinking than nonusers. ${ }^{67}$ Moreover, nonsmokers are less likely than smokers or ex-smokers to be in the high alcohol or high coffee drinking groups. ${ }^{67}$ Other researchers also report a significant positive correlation between smoking and drinking habits. ${ }^{68}$ In a review of studies on the interrelationships of tobacco, alcohol, and caffeine use, Istvan and Matarazzo reported that the use of alcohol and tobacco and the use of tobacco and caffeine are moderately to strongly related, whereas the use of alcohol and caffeine is only weakly related. ${ }^{69}$

Some studies have examined changes in the use of alcohol, tobacco, and caffeine during pregnancy, whereas others have explored pregnancy outcomes related to the use of at least two of these substances. More than one-half of 4,000 subjects in one study reported that their decreases in coffee drinking and smoking during pregnancy were largely attributed to nausea and vomiting. ${ }^{70}$ Consequently, some investigators believe that nausea and vomiting are adaptive responses that inhibit negative behaviors, and that that is why others find a positive association between nausea and vomiting and pregnancy outcomes. Little tested this hypothesis but found no support for this hypothesis. ${ }^{71}$ Alcohol use, smoking, and nausea and vomiting were all significantly related to lower birth weight. ${ }^{71}$

Some evidence of an interactive effect of maternal alcohol intake and smoking exists. ${ }^{72}$ One study found two-day-old infants had poorer performance on two operant learning tasks, head turning and sucking, if the mother drank alcohol and smoked or if the mother only smoked. Also, alcohol alone did not affect performance. ${ }^{72}$ Further, studies on alcohol, tobacco, and caffeine interaction are complicated by a number of other variables that may confound results. For example, in one study, cigarettes, alcohol, and marijuana would have each shown a greater influence on birth weight if examined separately rather than when these variables were examined together and with other confounding variables. ${ }^{25}$ Thus, the impact of individual behaviors may be relatively minor, but the impact of combined behaviors is more serious.

\section{CONCLUSION}

This review clearly indicates that smoking during pregnancy has negative consequences for the fetus. Heavy alcohol use (four to six drinks per day) also clearly has negative effects, whereas more moderate drinking has been seriously implicated in a number of studies. The effects of caffeine remain more questionable, but research evidence suggests enough concern to warrant caution.

That decreasing the use of either tobacco or alcohol at any point in pregnancy ameliorates some of the negative effects these substances have on fetal outcomes has been shown consistently. Consequently, nurses need to provide support and assistance for behavior change throughout pregnancy. Risky behaviors by women already at risk for other reasons, such as past history or socioeconomic status, warrant particular attention-especially considering the many studies in which variables such as age, nutritional status, and other health behaviors had an impact on pregnancy outcomes. However, health-care professionals also need to be sensitive to realistic options for change and recognize that any decrease in any of the risky behaviors could be significant and should be supported and acknowledged. Future re. 
I Nurses must be particularly alert to the presence of all risk factors and combinations of risky behaviors.

search may provide a clearer picture of the impact of the combined use of alcohol, tobacco, and caffeine on pregnancy outcomes, as well as the potential benefits of modifying use of these substances. Until then, health-care professionals can best serve their clients by helping them to understand the possible consequences of smoking and drinking (both alcohol and caffeine) during pregnancy and by encouraging and supporting minimal use if not total abstinence from these substances.

\section{ACKNOWLEDGMENT}

Work on this paper was supported by the National Center for Nursing Research, NIH, PHS, DHHS Grant Number 2 R01 NR 01207-03, L. Aaronson, principal investigator. The al1thors also wish to acknowledge Mary Jo Regan-Kubinski for her preliminary work reviewing the literature for this paper.

\section{REFERENCES}

1. Kleinman, J., and A. Kopstein. 1987. Smoking during pregnancy, 1967-1980. Am J Public Health. 77(7):82325.

2. Simpson, W.J. 1957. A preliminary report of cigarette smoking and the incidence of prematurity. Am J Obstet Gynecol. 73(4):808-15.

3. Mochizuki, M., T. Maruo, K. Masuko, and T, Ohtsu. 1984. Effects of smoking on fetoplacental-maternal system during pregnancy. Am J Obstet Gynecol. 149:41320.

4. Nilsen, S.T., N. Sagen, H.C. Kim, and P. Bergsjo. 1984. Smoking, hemoglobin levels, and birth weights in normal pregnancies. Am J Obstet Gynecol. 148:752-58.

5. Jensen, O.H., and O.P. Foss. 1981. Smoking and pregnancy. Acta Obstet Gynecol Scand. 60:177-81.

6. Ounsted, M., and A. Scott. 1982. Smoking during pregnancy. Acta Obstet Gynecol Scand. 61:367-71.

7. Fielding, J.E. 1978. Smoking and pregnancy. $N$ Engl $J$ Med. 798(6):337-39.

8. Meyer, M.B., B. S. Jonas, and J.A. Tonascia. 1976. Perinatal events associated with maternal smoking during pregnancy. Am J Epidemiol. 103(5):464-76.

9. Kleinman, J.C., and J.H. Matans. 1985. The effects of maternal smoking, physical stature, and educational attainment on the incidence of low birth weight. $A m J$ Epidemiol. 121(6):843-54.

10. Sexton, M. and J.R. Hebel. 1984. A clinical trial of change in maternal smoking and its effect on birth weight. JAMA. 251(7):911-15.

11. Institute of Medicine. 1984. Preventing low birthweight. Washington, D.C.: National Academy Press.
12. Koop, C. 1986. Smoking and pregnancy. Am Pharm. NS26(7):34-35.

13. Meyer, M.B., J.A. Tonascia, and C. Buck. 1975. The interrelationship of maternal smoking and increased perinatal mortality with other risk factors. Further analysis of the Ontario perinatal mortality study, 1960-1961. Am J Epidemiol. 100(6):443-52.

14. Cnattingius, S., O. Axelsson, G. Eklund, and G. Lindmark. 1985. Smoking, maternal age, and fetal growth. Obstet Gynecol. 66(4):449-52.

15. Andersen, K.V., and N. Hermann. 1984. Placenta flow reduction in pregnant smokers. Acta Obstet Gynecol Scand. 63:707-09.

16. Boomer, A.L., and B.L. Christensen. 1982. Antepartum hematocrit, maternal smoking and birth weight. $J$ Reprod Med. 27(7):385-88.

17. Van Der Veen, F., and H. Fox. 1982. The effects of cigarette smoking on the human placenta: A light and electron microscopic study. Placenta. 3:243-56.

18. Deibel, P. 1980. Effects of cigarette smoking on maternal nutrition and the fetus. J Obstet Gynecol Neonatal Nurs. 9(6):333-36.

19. Rush, D. 1976. Cigarette smoking during pregnancy: The relationship with depressed weight gain and birthweight. An updated report. In Birth Defects: Risks and Exposures, ed. Kelly, S., E.B. Hook, D.T. Janevich, and I.H. Parker. 161-72. New York: Academic Press, Inc.

20. Haworth, J.C., J.J. Ellestad-Sayed, J. King, and L.A. Dilling. 1980. Relation of maternal cigarette smoking, obesity, and energy consumption to infant size. Am J Obstet Gynecol. 138(8):1185-89.

21. Papoz, L., E. Eschwege, G. Pequignot, J. Barrat, and D. Schwartz. 1982. Maternal smoking and birth weight in relation to dietary habits. Am J Obstet Gynecol 142:87076.

22. Rush, D., A. Kristal, W. Blanc, et al. 1986. The effects of maternal cigarette smoking on placental morphology, hisomorphometry, and biochemistry. Am $J$ Perinatol. 3(3):263-72.

23. McIntosh, I.D. 1984. Smoking and pregnancy: Attributable risks and public health implications. Can J Public Health 75(2):141-48.

24. Goldstein, H. 1977. Smoking in pregnancy: Some notes on the statistical controversy. British Journal of Preventive and Social Medicine. 31(1):13-17.

25. Hingson, R., J.B. Gould, S. Morelock, et al. 1982. Maternal cigarette smoking, psychoactive substance use, and infant Apgar scores. Am J Obst Gynecol 144(8):959-66.

26. Christianson, R.E. 1980. The relationship between maternal smoking and the incidence of congenital anomalies. Am J Epidemiol. 112(5):684-95.

27. Naeye, R.L., and E.C. Peters. 1984. Mental development of children whose mothers smoked during pregnancy. Obstet Gynecol. 645:601-07.

28. Garn, S.M., M. Johnston, S.A. Ridella, and A.S. Petzold. 1981. Effect of maternal cigarette smoking on apgar scores. Am J Dis Child. 135:503-06.

29. Werler, M.M., B.R. Pober, and L.B. Holmes. 1985. Smoking and pregnancy. Teratology. 32:473-81.

30. Ericson, A., B. Kallen, and P. Westerholm. 1979. Cigarette smoking as an etiologic factor in cleft lip and palate. Am J Obstet Gynecol. 135(3):348-51. 
31. Khoury, M., A. Weinstein, S. Panny, et al. 1987. Maternal cigarette smoking and oral clefts: A population based study. Am J Public Health. 77(5):623-25.

32. Streissguth, A.P., D.C. Martin, H.M. Barr, B.M. Sandman, G.L. Kirchner, and B.L. Darby. 1984. Intrauterine alcohol and nicotine exposure: Attention and reaction time in 4-year-old children. Developmental Psychology. 20(4):533-41.

33. Schell, L.M., J.H. Relethford, and D.C. Hodges. 1986. Cigarette use during pregnancy and anthropometry of offspring 6-11 years of age. Hum Biol. 58(3):407-20.

34. Stjernfeldt, M., K. Berglund, J. Lindstein, and J. Ludvigsson. 1986. Maternal smoking during pregnancy and risk of childhood cancer. Lancet. 1(8494):1350-52.

35. Mills, J.L., B.I. Graubard, E.E. Harley, G.G. Rhoads, and H.W. Berendes. 1984. Maternal alcohol consumption and birth weight: How much drinking during pregnancy is safe? JAMA. 252(14):1875-79.

36. Little, R.E. 1977. Moderate alcohol use during pregnancy and decreased infant birth weight. $A m J$ Public Health. 67(12):1154-56.

37. Tennes, K., and C. Blackard. 1980. Maternal alcohol consumption, birth weight, and minor physical anomalies. Am J Obstet Gynecol. 138:774-80.

38. Russell, M. 1977. Intrauterine growth in infants born to women with alcohol-related psychiatric diagnoses. $A l$ coholism: Clinical \& Experimental Research. 1(3):225-31.

39. Barr, H.M., A.P. Streissguth, D.C. Martin, and C.S. Herman. 1984. Infant size at 8 months of age: Relationship to maternal use of alcohol, nicotine, and caffeine during pregnancy. Pediatrics. 74(3):336-41.

40. Zuckerman, B.S., and R. Hingson. 1986. Alcohol consumption during pregnancy: A critical review. Dev Med Child Neurol. 28:649-61.

41. Weiner, L., and H.L. Rosett. 1985. Alcohol effects on pregnancy: The experience at Boston City Hospital. Currents. 1(1):4-7.

42. Hatfield, D. 1986. Is social drinking during pregnancy harmless? Adv Alcohol Subst Abuse. 5(1-2):221-26.

43. Ouellette, E.M., and H.L. Rosett. 1976. A pilot prospective study of the fetal alcohol syndrome at the Boston City Hospital. Part II: The Infants. Ann NY Acad Sci. 123-29.

44. Kaminski, M., C. Rumeau, and D. Schwartz. 1978. Alcohol consumption in pregnant women and the outcome of pregnancy. Alcoholism: Clinical \& Experimental Research. 2(2):155-63.

45. Randall, C.L., W.J. Taylor, and D.W. Walker. 1977. Ethanol-induced malformations in mice. Alcoholism: Clinical \& Experimental Research. 1(3):219-24.

46. Weinberg, J. 1985. Effects of ethanol and maternal nutritional status on fetal development. Alcoholism: Clinical \& Experimental Research. 9(1):49-55.

47. Clarren, S.K., and D.W. Smith. 1978. The fetal alcohol syndrome. $N$ Engl J Med. 298(19):1063-67.

48. Bock, J. 1979. Closeup on fetal alcohol syndrome. The Canadian Nurse. 75:10.

49. Abel, E.L. 1984. Prenatal effects of alcohol. Drug Alcohol Depend. 14:1-10.

50. Streissguth, A.P. 1978. Fetal alcohol syndrome: An epidemiologic perspective. Am J Epidemiol. 107(6):46778.

51. Poskett, E.M.E. 1984. Fetal alcohol syndrome. Alcohol Alcohol. 19(2):159-65.
52. Murray-Lyon, I.M. 1985. Alcohol and fetal damage. $A_{t}$ cohol Alcohol. 20(2):185-88.

53. Smith, D.W., K.L. Jones, and J. W. Hanson. 1976. Per spectives on the cause and frequency of the fetal alco hol syndrome. Ann NY Acad Sci. 273:138-39.

54. Landesman-Dwyer, S., L.S. Keller, and A.P. Streissguth 1978. Naturalistic observations of newborns: Effects o maternal alcohol intake. Alcoholism: Clinical \& Experi mental Research. 2(2):171-77.

55. Sander, L.W., P.A. Snyder, H.L. Rosett, A. Lee, J.B Gould, and E. Ouellette. 1977. Effects of alcohol intake during pregnancy on newborn state regulation: A pro gress report. Alcoholism: Clinical \& Experimental $R e$ search. 1(3):233-41.

56. Oullette, E.M., H.L. Rosett, N.P. Rosman, and L. Weiner. 1977. Adverse effects of offspring of maternal alcohol abuse during pregnancy. $N$ Engl J Med. 297:528-30.

57. Marbury, M.C., S. Linn, R. Monson, S. Schoenbaum, P.G. Stubblefield, and K.J. Ryan. 1983. The association of alcohol consumption and outcome of pregnancy. Am d Public Health. 73(10):1165-68.

58. Blume, S.B. 1986. Is social drinking during pregnancy harmless? There is reason to think not. Adv Alcoho, Subst Abuse. 5(1-2):209-19.

59. Groisser, D.S., P. Rosso, and M. Winick. 1982. Coffee consumption during pregnancy: Subsequent behavioral abnormalities of the offspring. J Nutr. 112:829-32.

60. U.S. Food and Drug Administration. 1980. Drug Bulletin: Caffeine and Pregnancy. 10(3):17-20.

61. Brooten, D., and C.H. Jordan. 1983. Caffeine and pregnancy: A research review and recommendations for clinical practice. J Obstet Gynecol Neonatal Nurs. 12(3):190-95.

62. Weatherbee, P.S., L.K. Olsen, and J.R. Lodge. 1977. Caffeine and pregnancy. Postgraduate Medicine. 62(3):6469.

63. Linn, S., S.C. Schoenbaum, R.R. Monson, B. Rosner, P.G. Stubblefield, and K.J. Ryan. 1982. No association between coffee consumption and adverse outcomes of pregnancy. $N$ Engl J Med. 306(3):141-45.

64. Kurppa, K., P.C. Holmberg, E. Kuosma, and L. Saxen. 1983. Coffee consumption during pregnancy and selected congenital malformations: A nationwide casecontrol study. Am J Public Health. 73(12):1397-99.

65. Morris, M.B., and L. Weinstein. 1981. Caffeine and the fetus: Is trouble brewing? Am $J$ Obstet Gynecol. 140(6):607-10.

66. Aldridge, A., J. Bailey, and A.H. Neims. 1981. The disposition of caffeine during and after pregnancy. Seminars in Perinatology. 5(4):310-14.

67. Carmody, R.P., C.S. Brischetto, J.D. Matarazzo, and R.P. O'Donnell. 1985. Co-occurent use of cigarettes, alcohol, and coffee in healthy, community-living men and women. Health Psychol. 4(4):323-35.

68. Craig, T.J., and P.A. Van Natta. 1977. The association of smoking and drinking habits in a community sample. $J$ Stud Alcohol. 38(7):1434-39.

69. Istvan, J., and J.D. Matarazzo. 1984. Tobacco, alcohol and caffeine use: A review of their interrelationships. Psychol Bull. 95(2):301-26.

70. Hook, E.B. 1976. Changes in tobacco smoking and ingestion of alcohol and caffeinated beverages during early pregnancy: Are these consequences, in part, of 
feto-protective mechanisms diminishing maternal exposure to embryotoxins? In Birth Defects: Risks \& Consequences, ed. Kelly, S., E.B. Hook, D.T. Janevich, and I.H. Porter, New York: Academic Press.

71. Little, R.E. 1980. Maternal alcohol and tobacco use and nausea and vomiting during pregnancy: Relation to infant birthweight. Acta Obstet Gynecol Scand. 59:495-97.

72. Martin, J., D.C. Martin, C.A. Lund, and A.P. Streissguth. 1977. Maternal alcohol ingestion and cigarette smoking and their effects on newborn conditioning. Alcoholism: Clinical \& Experimental Research. 1(3):243-47.
Address for correspondence: Lauren S. Aaronson, University of Kansas School of Nursing, 39th and Rainbow Blvd., Kansas City, KS 66103.

Lauren S. Aaronson is associate dean for research at the University of Kansas School of Nursing in Kansas City, Kansas. Dr. Aaronson is a member of the Council of Nurse Researchers, the Midwest Nursing Research Society, and Sigma Theta Tau (Rho Chapter).

Carol L. Macnee is a research assistant at the University of Michigan's School of Nursing in Ann Arbor, Michigan. Ms. Macnee is a member of the American Nurses' Association, Sigma Theta Tau (Rho Chapter), and the Midwest Nursing Research Society.

\title{
Are you providing couplet care? \\ Do you work in a health-care setting where couplet care is being planned for the near future?
}

\section{Would you like your institution to offer couplet care?}

\author{
Then Mother-Baby Care is must reading for you \\ philosophy, \\ benefits, \\ preparation of the organizational system. \\ integration and change theory, and \\ cross-training \\ as they relate to converting to couplet care.
}

Mother-Baby Care, NAACOG's 13-page practice resource, discusses

Order Mother-Baby Care today and help shape the future of couplet care in your work setting

To receive your copy of Mother-Baby Care, simply fill out the order form below and return it, with payment, to NAACOG, 409 12th St, S.W., Washington, DC 20024-2191. Allow 8 weeks for delivery. Call 1 (202) 863-2464 with any questions.

HURRY! I can't wait to receive my own copy of Mother-Baby Care.

send me copy(ies) at $\$ 3.00$ each, $\$ 15.00$ for six Less $20 \%$ member discount $6 \%$ sales tax for DC residents $5 \%$ for MD residents Postage Up to $\$ 5.00, \$ 0.50$

Up to $\$ 10, \$ 1.00$

Up to $\$ 25, \$ 2.00$

Up to $\$ 50, \$ 3.00$

Please Print Neatly

Name

Address

Member ID \#
ALL ORDERS MUST BE

PREPAID. Make check payable

to NAACOG. Mail to

NAACOC Publications,

409 12th St., S.W.,

Washington, DC 20024-2191.

Prices effective through 12/31/89 